\title{
An Atom Probe Tomography Study of Ni-Cr-Al-Ti High Temperature Oxidation
}

\author{
Talia L. Barth ${ }^{1}$ and Emmanuelle A. Marquis ${ }^{1}$ \\ 1. University of Michigan Department of Materials Science and Engineering, Ann Arbor, MI, USA.
}

High-strength nickel alloys that are used in high-temperature applications, such as in gas turbine engines, are susceptible to oxidation under the extreme conditions they experience. To increase their resistance to oxidation, elements such as chromium and aluminum are added in order to form dense, protective oxides on the alloy surface that protect the underlying metal from further oxidation. Additional dopants such as yttrium are typically added to improve the lifetime of these protective scales by decreasing their growth rate and increasing the scale adhesion, though the mechanisms of this effect as well as differences in oxidation behavior with various elements is not always clearly understood. The steady-state oxidation kinetics of the Ni-Cr-Al system is well-documented [1], but studies of dopant effects generally involve more complex superalloys with a wide range of additional elements, making it difficult to isolate the effect of each element addition. Particularly, the effect of titanium on the oxidation behavior of these alloys is unclear. Several studies [2] have shown that Ti additions are detrimental to scale lifetime and increase the oxide growth rate. Others [3] claim that Ti additions improve the scale adhesion. For this study, model $\mathrm{Ni}-\mathrm{Cr}-\mathrm{Al}$ alloys with varied amounts of $\mathrm{Ti}$ were oxidized at high temperature in order to investigate the effect of titanium on oxidation behavior.

Three different alloys, Ni-26Cr-10Al, Ni-26Cr-10Al-0.6Ti, and Ni-Cr-10Al-1.7Ti (at\%), were ground with $\mathrm{SiC}$ paper to 1200 grit and then polished using diamond suspension to $1 \mathrm{um}$. They were then oxidized in a $20 \% \mathrm{O} 2$-Ar atmosphere at $1000{ }^{\circ} \mathrm{C}$ for 30 minutes, 2 hours, and 8 hours. After oxidation, electron transparent cross-section lamellae of the oxide scales for Transmission Electron Microscopy (TEM) and Scanning TEM, and needle-shaped specimens for atom probe tomography (APT) analysis were prepared using a Focused Ion Beam (FIB) lift out technique on a FEI Helios 650 Nanolab SEM/FIB. A JEOL 2010F Analytical Electron Microscope with an attached EDAX detector was used for TEM imaging and energy dispersive x-ray spectroscopy analysis, and a Cameca LEAP 4000X HR instrument was used for detailed composition analysis.

Oxidation of the titanium-free alloy for 2 hours produced a layered oxide scale consisting of $\mathrm{NiO}$ islands embedded in Ni-Cr oxide (spinel), a continuous $\mathrm{Cr}_{2} \mathrm{O}_{3}$ layer, and $\mathrm{Al}_{2} \mathrm{O}_{3}$ fingers underneath, with Ni-rich grains between the chromia and alumina. In contrast, the alloys with titanium exhibited much thinner, more uniform scales, shown in Figure 1. In particular, the alumina "fingers" had grown into a flat, continuous layer. In the Ti-containing alloy, the chromia layer was much thinner relative to the mixed $\mathrm{Ni}-\mathrm{Cr}$ oxide layer. APT specimens were prepared within the scale to capture both $\mathrm{Ni}-\mathrm{Cr}$ oxide (green) and chromia (pink) grains, which can be seen in the APT reconstruction shown in Figure 2a. The APT reconstruction also reveals small Ti-rich oxide particles in the chromia grains, and Ti segregation on the grain boundaries, which can be seen in Figure 2b. Following a detailed analysis of the differences and evolution during oxidation exposures, the roles and effects of Ti additions will be discussed [4].

References:

[1] C Giggins and F Pettit, Journal of the Electrochemical Society 118 (1971), p. 1782.

[2] B Pint et al, TMS Superalloy Conference Proceedings (2000) p. 629. 
[3] D Clemens, W Quadakkers, and L Singheiser, Electrochemical Society Proceedings (1998), p. 134. [4] The authors acknowledge funding from the National Science Foundation under grant DMR-1352157 and technical support from the Michigan Center for Materials Characterization.

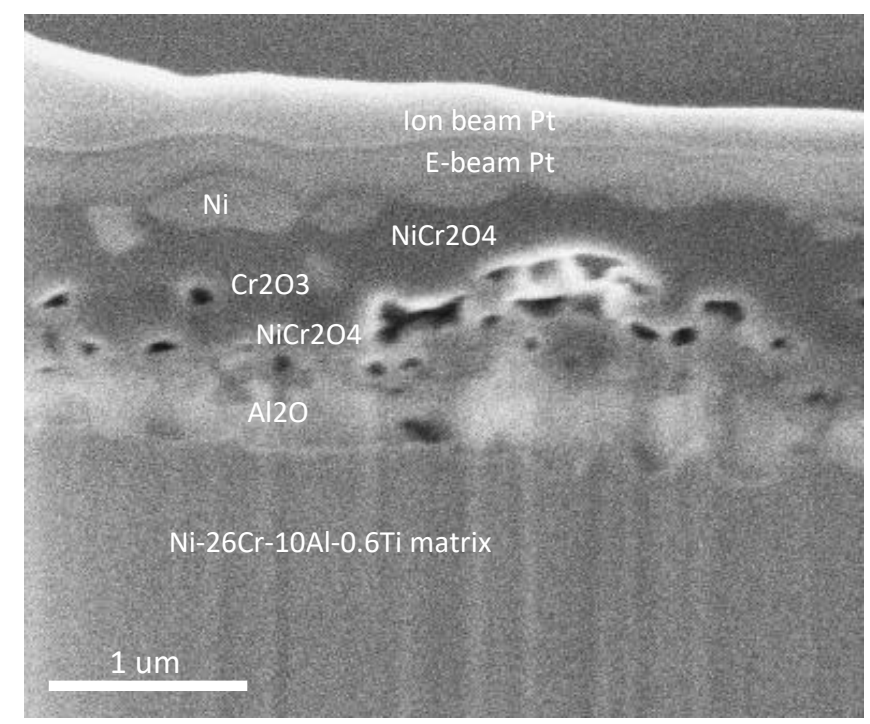

Figure 1. SEM image of a FIB-prepared cross-section of a Ni-26Cr-10Al-0.6Ti alloy oxidized for 2 hours at $1000^{\circ} \mathrm{C}$. The oxide phases labelled on the image were determined using qualitative EDS mapping.

(a)

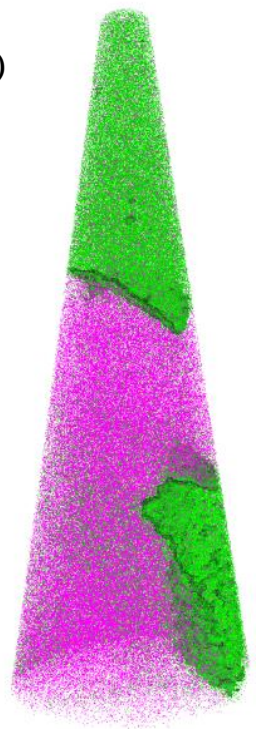

(b)

$220 \mathrm{~nm}$

Figure 2. (a) APT reconstruction of a portion of the scale from a Ni-26Cr-10Al-0.6Ti alloy oxidized for 2 hours at $1000{ }^{\circ} \mathrm{C}$. Nickel ions are shown in green and chromium ions are shown in pink. (b) Ti, TiO, and $\mathrm{TiO}_{2}$ ions are shown in blue. 